\title{
Defect Visualization and Effective Data Compression Techniques for Infrared Pulsed Thermography
}

\author{
Ajay Rawat, Sharath D, M. Menaka, and B. Venkatraman \\ Indira Gandhi Centre for Atomic Research, Kalpakkam 603 102, India \\ ajayrawat@igcar.gov.in
}

In Pulsed thermography experiment depending on the camera specification one deal with a 3D data of large size, extracting the relevant information from the experimental data can be quite tedious. For doing any inferential and quantitative analysis from the thermography data in a semi-automated and automated manner it is necessary that the dimension of the experimental data is reduced without losing much of the information. Higher order statistics, Thermal signal reconstruction and Principle component thermography are some of the few data compression techniques which we'll discuss in this paper and provide some comparative results.

Keywords: Signal Processing, Pulsed Thermography, Semi-automated Processing. 